\title{
¿DE QUÉ SE HABLA EN LOS FOROS DE INTERNET PARA PROBLEMAS DE SALUD? ANÁLISIS DEL CONTENIDO EN FUNCIÓN DE LA ENFERMEDAD Y EL SEXO \\ WHAT ARE PEOPLE TALKING ABOUT ON HEALTH-RELATED INTERNET FORUMS? CONTENT ANALYSIS BY ILLNESS AND GENDER
}

\author{
RAQUEL SURIÁ y MARINA BELÉNDEZ \\ Departamento de Comunicación y Psicología Social. Universidad de Alicante. \\ Autora para correspondencia: Marina Beléndez Vázquez. E-mail: marina.belendez@ua.es
}

\section{Resumen}

En los últimos años, Internet ha posibilitado la aparición y consolidación de nuevas formas de comunicación interpersonal. Entre ellas destaca la establecida entre los participantes en los foros de discusión sobre problemas relacionados con la salud. Una de las líneas de investigación más fructíferas se dirige al análisis del contenido de los mensajes intercambiados entre los participantes.

El presente estudio analiza la frecuencia de los tópicos intercambiados en las contribuciones de los usuarios de foros de discusión para problemas de salud en función del sexo y de la categoría diagnóstica.

Se recopilaron 756 mensajes de cuatro foros relacionados con problemas de salud (diabetes, fibromialgia, esclerosis y asma). Los mensajes se analizaron mediante un código que incluyó 15 categorías de análisis agrupadas en cuatro dimensiones: intercambio de apoyo social, enfermedad y tratamiento, aspectos interpersonales y emociones.

Los resultados indican que las referencias a apoyo informativo y práctico eran más frecuentes en el foro de diabetes. Las expresiones de emociones positivas (optimismo y gratitud) se encontraban más en el foro de fibromialgia. Las

\begin{abstract}
In recent years, Internet has made possible the emergence and consolidation of a new form of interpersonal communication such as the discussion among members of health related bulletin boards or discussion forums. One of the more fruitful research work is aimed to analyze the content of the exchanged messages among participants.
\end{abstract}

The present work analyzes the frequency of the topics in the messages that are exchanged among users of internet discussion forums for health problems by sex and illness.

A total of 756 postings of participants in four Spanish-speaking internet bulletin boards were analyzed (diabetes, fibromyalgia, sclerosis and asthma). Each posting was analyzed for the presence or absence of 15 coding categories subsumed under four broad categories (social support, illness and treatment issues, interpersonal issues and expression of emotions).

Postings regarding giving informational support and instrumental support were more frequent at diabetes discussion board. Expressions of positive emotions (optimism and gratitude) were predominately found on fibromyalgia boards. Differences by sex were only found in some categories and in some forums. 
diferencias en la frecuencia de los temas de los mensajes en función del sexo aparecen sólo en algunos tópicos y, únicamente, en los foros de algunas enfermedades.

\section{Palabras Clave}

Foros de internet, apoyo social, análisis de contenido, enfermedad.

\section{Introducción}

Los foros de discusión de Internet se han consolidado como uno de los principales canales para el intercambio de información y apoyo en cuestiones referentes a problemas de salud (Demiris, 2006). Este nuevo fenómeno ha suscitado el interés de investigadores de disciplinas diversas y en las últimas dos décadas el estudio de los foros de discusión, las comunidades virtuales o los grupos de ayuda mutua online constituye una fructífera área de trabajo para estudiosos de la comunicación, de las ciencias de la salud o de la psicología.

Junto al interés indiscutible que suscita el conocimiento de la naturaleza de los contenidos intercambiados en estos foros, otra razón que explica el rápido avance y desarrollo de esta área de estudio es la facilidad en el acceso a los datos objeto de estudio. Así, las propias características del canal de comunicación, en formato escrito, con acceso instantáneo desde cualquier ordenador personal y con disponibilidad de archivos de mensajes, facilitan considerablemente la labor investigadora.

El análisis de las contribuciones a los foros online de discusión constituye una forma alternativa y valiosa de aproximarse al estudio de la vivencia de la enfermedad. El contexto virtual en el que se desarrolla esta comunicación, caracterizado por el anonimato y la consiguiente ausencia de señales visuales promueve la revelación personal (Barak y Gluck-Ofri, 2007). Este efecto puede explicarse por el fenómeno de «desindividuación», que en el contexto virtual se traduce en una forma de interacción hiperpersonal, que facilita la expresión abierta de emociones (Moral, Canto y Gómez-Jacinto, 2004; Walther y Boyd, 2002).

\section{Key Words}

Internet forums, social support, content analysis, illness.

Por tanto, el análisis de estos mensajes nos facilita información sobre las necesidades, creencias, preocupaciones y preferencias de pacientes y familiares, que son de especial interés para aquellos interesados en el estudio psicosocial de la experiencia de enfermedad (Davison y Pennebaker, 1997).

Las estrategias que con más frecuencia se han utilizando para el estudio del contenido de las contribuciones de los participantes de los foros virtuales han sido las técnicas de análisis de contenido (Weare y Lin, 2000). Estas técnicas nos ofrecen la posibilidad de sistematizar y codificar los mensajes con el objetivo de identificar cuáles son los tópicos más frecuentes sobre los que se intercambia información entre los usuarios de este tipo de foros. La mayoría de los estudios dirigidos al análisis del contenido de los mensajes intercambiados en estos foros de discusión online y grupos de auto-ayuda virtuales coinciden en destacar que los temas estrella sobre los que se «escribe» pueden agruparse en torno a tres grandes aspectos: intercambio de apoyo social, experiencias de enfermedad y revelaciones personales (Anderberg, 2007; Bar-Lev, 2008; Blank y Adams-Blodnieks, 2007; Coulson, 2005; Finn, 1999; Ginossar, 2008; Lasker, Sogolow y Sharim, 2005; Perron, 2002; Salem, Bogat y Reid, 2004; Schultz, Stava, Beck y Vassilopoulou-Sellin, 2003; Sharf, 1999; Sullivan, 2003; Winzelberg, 1997; Winefield, 2005).

Aunque la mayoría de los estudios tienden a coincidir en estos tópicos como los temas centrales del contenido de los mensajes intercambiados, parecen existir diferencias en la frecuencia con que se abordan unos u otros en función del problema de salud al que se dirige el foro (Mehl y Pennebaker, 2003). Mientras en al- 
gunos foros, como los de anorexia, cáncer o esclerosis, se detecta una tendencia a intercambiar mensajes que abordan en mayor medida aspectos personales y de revelación emocional (Winzelberg, 1997), en otros foros, como los de diabetes, parecen abundar los mensajes que inciden más en el intercambio de apoyo informativo (Ravert, Hancock e Ingersoll, 2004). Parece, pues, que las diferencias encontradas podrían deberse a la existencia de patrones propios de expresión en función del problema de salud (Davison y Pennebaker, 1997). Así, la naturaleza de cada enfermedad (crónica, degenerativa, de curso impredecible, de origen desconocido, etc.) podría determinar el modo en que las personas afectadas, ya sean pacientes como familiares, escriben acerca de su vivencia.

Sin embargo, aunque recientemente se han llevado a cabo algunos estudios comparativos con la inclusión de foros sobre distintos problemas de salud (Macias, Stavchansky y Smith, 2005; Van Uden-Kraan et al., 2008), la mayoría de ellos se han centrado en el estudio de foros dedicados a problemas de salud específicos. Por tanto, resulta difícil concluir si las diferencias en el tipo de contenidos que se intercambian en los foros virtuales vienen determinadas por las peculiaridades de cada problema de salud o, más bien, por la disparidad de procedimientos de categorización y codificación de los mensajes.

Además de las preferencias por unos temas en función del tipo de problema de salud también se sugiere la posibilidad de que existan diferencias en el contenido de los mensajes en función del sexo de los participantes. Los defensores de esta hipótesis coinciden en indicar que las mujeres tienden a expresar más apoyo emocional y empatía en sus contribuciones online en comparación con los hombres, más proclives a dirigir mensajes de contenido puramente informativo (Klemm, Hurst, Dearholt y Trone, 1999; Seale, Ziebland y Charteris-Black, 2006). Este patrón diferencial de comunicación se ha observado previamente en el estudio de grupos de apoyo cara a cara, sobre todo al comparar grupos de apoyo para pacientes de cáncer (Gray, Fitch, Davis y Phillips, 1996). No obstante, otros estudios sugieren la hipótesis que propone que la naturaleza del contexto online mitigaría las diferencias de género observadas en la comunicación tradicional; por tanto, el anonimato propio de este tipo de interacción favorecería la similitud en el patrón de comunicación entre hombres y mujeres. Recientemente, Mo, Malik y Coulson (2009), tras la revisión sistemática de una docena de estudios sobre grupos virtuales relacionados con cuestiones de salud y género, concluyen la existencia tanto de similitudes como diferencias. Así, los resultados de los estudios que comparan mensajes de foros sobre problemas de salud masculinos (p. ej. cáncer de próstata) con los enviados a foros sobre problemas femeninos (p. ej. cáncer de mama) revelan diferencias, mientras que aquellos estudios sobre foros o grupos mixtos han encontrado que los patrones de comunicación de hombres y mujeres son similares.

Aunque existe evidencia de que la participación en foros para problemas relacionados con la salud constituye una de las actividades preferidas para quienes se acercan a la red por cuestiones relacionadas con la salud (Cline y Haynes, 2001) y, en concreto, entre usuarios de nuestro país, son escasos los estudios dirigidos a analizar los contenidos que se intercambian en foros en lengua española. Asimismo, la mayoría de los estudios sobre análisis de contenido de los foros relacionados con salud se han centrado en el análisis de una única enfermedad. Además, como revisamos más arriba, la cuestión de la tendencia a determinados contenidos en los mensajes sobre otros en función del sexo continúa abierta. Por tanto, y con el objetivo de profundizar más en esta línea de investigación, proponemos este estudio.

Los objetivos de este trabajo son, en primer lugar, identificar los tópicos o temas más frecuentes de los mensajes intercambiados entre los participantes en diversos foros de internet sobre problemas de salud en función del grupo diagnóstico y, en segundo lugar, en función del sexo de los participantes para cada uno de esos mismos grupos.

\section{Método}

\section{Muestra}

El estudio se centra en al análisis de los mensajes intercambiados entre participantes de 
foros de internet de cuatro enfermedades: fibromialgia, asma, esclerosis y diabetes. Se seleccionaron enfermedades que fueran diferentes en aspectos como la tasa de prevalencia, el curso o la disponibilidad de tratamientos efectivos. Siguiendo a Davidson, Pennebaker y Dickerson, (2000), el proceso de selección de trastornos se guió por un criterio práctico, más que teórico. De este modo, se persiguió el objetivo de abarcar un rango amplio de problemas de salud con el fin de poder incluir trastornos variados en sus tasas de prevalencia, condiciones potencialmente mortales, así como aquellas con un componente psicológico y comportamental importante.

Se seleccionaron varios foros que tuvieran un nivel de actividad adecuado, indicado por un número de mensajes archivados superior a 50 mensajes al mes y que fueran de acceso libre. Se recopilaron todos los mensajes enviados a los foros durante un periodo de tiempo de dos meses y medio. De este modo, se seleccionó un total de 756 mensajes: fibromialgia $(n=207)$, asma $(n=131)$, esclerosis $(n=180)$ y diabetes $(n=238)$.

\section{Código de análisis}

Se elaboró un código que incluyó 15 categorías de análisis agrupadas en cuatro dimensiones generales que incluían los tópicos o temas analizados en trabajos previos (Lasker, Sogolow y Sharim, 2005; Perron, 2002): intercambio de apoyo social, aspectos relativos a la enfermedad y el tratamiento, aspectos interpersonales y emociones. Así, las 15 categorías finales de análisis fueron: intercambio de apoyo social (6 categorías): contenido (apoyo emocional, instrumental o informativo) y, a la vez, dirección (ofrecimiento o demanda), enfermedad y tratamiento (3 categorías): síntomas físicos, síntomas psicológicos y cuestiones relacionadas con el tratamiento, aspectos interpersonales (3 categorías): temas relacionados con la familia, los amigos $\mathrm{u}$ otras personas y emociones ( 3 categorías): optimismo, tristeza y gratitud.

La unidad de análisis fue el mensaje. Así, cada mensaje se codificaba según la presencia (1) o ausencia (0) de cada tópico o tema, es decir, un mensaje podía recibir múltiples códigos.
Por último, para identificar el sexo de los participantes se realizó un seguimiento a través de sus «nicks» y la lectura del mensaje.

\section{Procedimiento}

El análisis de los mensajes fue realizado por una de las autoras (R.S). Otro investigador analizó un 20\% de los mensajes (152) y se calculó la fiabilidad inter-jueces mediante el índice Kappa de Cohen. Se alcanzó un índice de acuerdo mayor de 0.75 , lo que revela un valor adecuado (Rourke, Anderson, Garrison y Archer, 2001).

\section{Resultados}

De los 756 mensajes analizados, más del $60 \%$ fueron enviados por mujeres. Excepto en el foro de esclerosis, en el que un $62.2 \%$ de los mensajes los enviaron varones, en el resto de foros la mayoría de los mensajes proceden de mujeres, $\chi^{2}(1, \mathrm{~N}=756)=55.25, \mathrm{p}=0.001$ (ver figura 1).

Respecto a las cuatro dimensiones generales analizadas, los resultados mostraron que la mayoría de los mensajes aludían a aspectos relacionados con el intercambio de apoyo social $(95.6 \%)$, a cuestiones referidas a la enfermedad o el tratamiento $(81.9 \%)$ y a aspectos interpersonales $(68 \%)$. En menor medida, los mensajes

Figura 1. Frecuencia de mensajes enviados por mujeres y por varones según enfermedad.

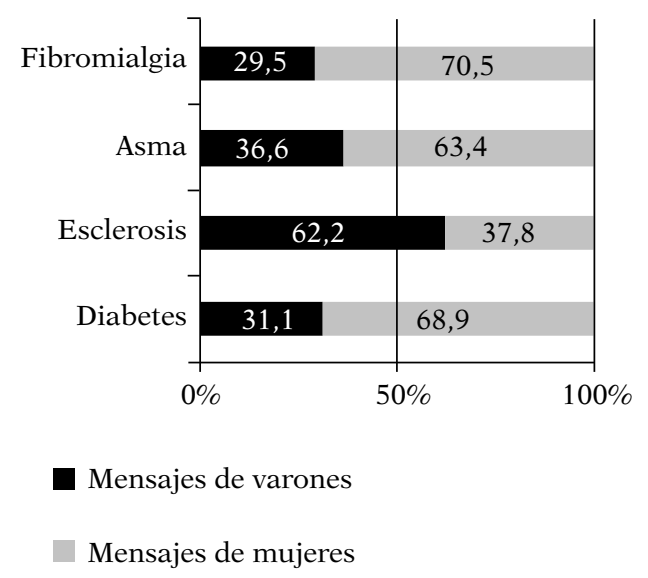


Tabla 1. Porcentaje de cada uno de los temas en los mensajes por enfermedades.

\begin{tabular}{|c|c|c|c|c|c|}
\hline & Total & Fibromialgia & Asma & Esclerosis & Diabetes \\
\hline \multicolumn{6}{|l|}{ Ароуо } \\
\hline Apoyo Informativo-Ofrece ${ }^{* * *}$ & 55.2 & 40.1 & 42 & 60 & 71.8 \\
\hline Apoyo Práctico-Ofrece ${ }^{* *}$ & 32 & 23.7 & 21.4 & 34.4 & 43.3 \\
\hline Apoyo Emocional-Ofrece $e^{* *}$ & 21.3 & 39.6 & 3.8 & 23.9 & 13 \\
\hline Apoyo Informativo-Demanda** & 20.1 & 15.9 & 45.8 & 9.4 & 17.6 \\
\hline Apoyo Práctico-Demanda** & 11.8 & 6.8 & 22.1 & 11.1 & 10.5 \\
\hline Apoyo Emocional-Demanda** & 11.6 & 20.8 & 6.9 & 11.7 & 6.7 \\
\hline \multicolumn{6}{|l|}{ Enfermedad } \\
\hline Sintomas físicos** & 51.1 & 39.1 & 45 & 58.3 & 59.2 \\
\hline Síntomas psicológicos $* *$ & 23.5 & 22.7 & 1.5 & 44.4 & 20.6 \\
\hline Tratamiento $* *$ & 76.3 & 64.3 & 87.8 & 71.1 & 84.5 \\
\hline \multicolumn{6}{|l|}{ Aspectos interpersonales } \\
\hline Familia** & 14.8 & 3.9 & 52.7 & 3.9 & 11.8 \\
\hline Amigos** & 16.3 & 34.8 & 3.1 & 17.8 & 6.3 \\
\hline Otros** & 37.6 & 45.9 & 14.5 & 15.6 & 59.7 \\
\hline \multicolumn{6}{|l|}{ Emociones } \\
\hline Optimismo** & 17.9 & 29.5 & 7.6 & 15 & 15.5 \\
\hline Tristeza* & 11.5 & 10.6 & 16.8 & 6.1 & 13.4 \\
\hline Gratitud $^{* * *}$ & 16.8 & 28.5 & 22.9 & 10 & 8.4 \\
\hline
\end{tabular}

$* \mathrm{p}<0.05 . * * \mathrm{p}<0.001$ para prueba $\chi^{2}$ comparando los grupos de enfermedad

explicitan emociones (30.6\%). Por temas específicos, se observa que el ofrecimiento de apoyo es más frecuente que la demanda del mismo en sus tres dimensiones, informativa, práctica y emocional, (ver tabla 1). Respecto a los temas específicos de la enfermedad, un porcentaje notable de los mensajes se refieren a cuestiones del tratamiento $(76.3 \%)$, mientras que, aproximadamente la mitad de las contribuciones aluden a síntomas físicos.

\section{Análisis de contenido: Frecuencias de temas según la enfermedad}

$\mathrm{Al}$ examinar los tópicos intercambiados en los mensajes, se observan diferencias significativas en la frecuencia de todos los temas según la enfermedad (ver tabla 1).

Respecto a los mensajes que demandan apoyo informativo, son los participantes del foro de asma (45.8\%) y de diabetes $(17.3 \%)$, los que más aluden a este tópico, $\chi^{2}(3, \mathrm{~N}=756)=69.71, \mathrm{p}<$
0.001. En cuanto a la demanda de apoyo práctico, destaca el grupo de asma, $\chi^{2}(3, \mathrm{~N}=756)=$ $19.16, \mathrm{p}<0.001$. Por su parte, son los foros de diabetes y esclerosis los que incluyen más ofrecimientos de apoyo informativo, $\chi^{2}(3, \mathrm{~N}=756)$ $=56.68, \mathrm{p}<.001, \mathrm{y}$ apoyo práctico, $\chi^{2}(3, \mathrm{~N}=756)$ $=27.79, \mathrm{p}<0.001)$.

La demanda de apoyo emocional aparece con más frecuencia en los foros de fibromialgia y esclerosis $\left.\chi^{2}(3, \mathrm{~N}=756)=25.02, \mathrm{p}<0.001\right)$. Consecuentemente, también son los mensajes de los foros de fibromialgia y, de nuevo, los del foro de esclerosis, los que contienen más referencias a ofrecimientos de apoyo emocional, $\chi^{2}$ $(3, \mathrm{~N}=756)=75.75, \mathrm{p}<0.001$.

En relación a los tópicos relativos a la enfermedad, son los mensajes del grupo de diabetes los que con mayor frecuencia mencionan sintomas físicos, $\chi^{2}(3, \mathrm{~N}=756)=23.87, \mathrm{p}<0.001, \mathrm{y}$, junto al de asma, aluden más al tratamiento, $\chi^{2}$ $(3, \mathrm{~N}=756)=37.63, \mathrm{p}=0.001$, mientras que en el foro de esclerosis se mencionan más cuestio- 
nes relativas a síntomas psicológicos, $\chi^{2}(3, \mathrm{~N}=$ 756) $=80.19, \mathrm{p}<0.001)$.

Respecto a las alusiones interpersonales de los mensajes, destacó el grupo de asma $(52.7 \%)$, por su mayor frecuencia de contenidos referentes a la familia, $\chi^{2}(3, \mathrm{~N}=756)=187.21, \mathrm{p}<$ 0.01 , y el de fibromialgia $(34.8 \%)$ por incluir más referencias a relaciones con amigos, $\chi^{2}(3$, $\mathrm{N}=756)=86.53, \mathrm{p}<0.001$.

Por último, son los mensajes del foro de fibromialgia los que incluyen más referencias a emociones positivas: optimismo, $\chi^{2}(3, \mathrm{~N}=756)=$ $30.23, \mathrm{p}<0.001$ y gratitud, $\chi^{2}(3, \mathrm{~N}=756)=$ 41.73, $\mathrm{p}<0.001$, mientras que las contribuciones al foro de esclerosis presentan menos alusiones a la tristeza, $\chi^{2}(3, \mathrm{~N}=756)=9.77, \mathrm{p}<0.05$.

\section{Análisis de contenido: Frecuencias de temas según el sexo}

Al examinar la frecuencia de los temas de los mensajes en función del sexo, las preferen- cias de mujeres o varones por unos temas se hacen patentes sólo en algunos tópicos y, únicamente, en los foros de algunas enfermedades. Así, no se hallaron diferencias significativas de género en la frecuencia de alusión a temas relativos a sintomas y tratamiento ni en el contenido emocional (expresiones de tristeza y optimismo) en ninguno de los foros de las cuatro enfermedades (ver tabla 2). Únicamente se observa una mayor frecuencia de expresiones de gratitud de los mensajes de mujeres en comparación con el de los varones en el foro de asma, $\chi^{2}(1, \mathrm{~N}=$ 131) $=4.64, \mathrm{p}<0.05$.

Las diferencias de género aparecen cuando se analizan la frecuencia de contenidos relativos al ofrecimiento de apoyo. En este sentido, se observó que los mensajes enviados por varones incluyeron más referencias de oferta de apoyo informativo que los de las mujeres en los foros de diabetes, $\chi^{2}(1, \mathrm{~N}=238)=11.37, \mathrm{p}<0.01 \mathrm{y}$ asma $\chi^{2}(1, \mathrm{~N}=131)=29.75, \mathrm{p}<0.001$. Del mismo modo, sólo en el foro de diabetes, los mensajes enviados por varones aluden más a ofertas de apoyo práctico, $\chi^{2}(1, \mathrm{~N}=238)=31.87, \mathrm{p}<0.001$.

Tabla 2. Porcentaje de cada uno de los temas en los mensajes en función del sexo (V, varón; M, mujer) para cada enfermedad.

\begin{tabular}{|c|c|c|c|c|c|c|c|c|}
\hline \multirow{2}{*}{ Apoyo } & \multicolumn{2}{|c|}{ Fibromialgia } & \multicolumn{2}{|c|}{ Asma } & \multicolumn{2}{|c|}{ Esclerosis } & \multicolumn{2}{|c|}{ Diabetes } \\
\hline & $\mathrm{V}$ & M & V & M & V & $\mathrm{M}$ & V & M \\
\hline Apoyo Informativo-Ofrece & 36.1 & 41.8 & 72.9 & 24.1 & 58 & 63.2 & 86.5 & 65.2 \\
\hline Apoyo Práctico-Ofrece & 24.6 & 23.3 & 33.3 & 14.5 & 31.3 & 39.7 & 70.3 & 31.3 \\
\hline Apoyo Emocional-Ofrece & 32.8 & 42.5 & 2.1 & 4.8 & 17 & 35.3 & 10.8 & 14 \\
\hline Apoyo Informativo-Demanda & 19.7 & 14.4 & 16.7 & 62.7 & 8.9 & 10.4 & 12.2 & 20.1 \\
\hline Apoyo Práctico-Demanda & 6.6 & 6.8 & 10.4 & 28.9 & 10.7 & 11.8 & 8.1 & 11.6 \\
\hline Apoyo Emocional-Demanda & 26.2 & 18.5 & 2.1 & 9.6 & 14.3 & 7.4 & 1.4 & 9.1 \\
\hline \multicolumn{9}{|l|}{ Enfermedad } \\
\hline Síntomas físicos & 41 & 38.4 & 37.5 & 49.4 & 57.1 & 60.3 & 60.8 & 58.5 \\
\hline Síntomas psicológicos & 29.5 & 19.9 & 4.2 & 0.0 & 43.8 & 45.6 & 21.6 & 20.1 \\
\hline Tratamiento & 70.5 & 61.6 & 89.6 & 86.7 & 72.3 & 69.1 & 85.1 & 84.1 \\
\hline \multicolumn{9}{|l|}{ Aspectos interpersonales } \\
\hline Familia & 6.6 & 2.7 & 22.9 & 69.9 & 4.5 & 2.9 & 9.5 & 12.8 \\
\hline Amigos & 26.2 & 38.4 & 0.0 & 4.8 & 14.3 & 23.5 & 6.8 & 6.1 \\
\hline Otros & 52.5 & 43.2 & 18.8 & 12.0 & 22.3 & 4.4 & 63.5 & 57.9 \\
\hline \multicolumn{9}{|l|}{ Emociones } \\
\hline Optimismo & 23.3 & 32.2 & 2.1 & 10.8 & 12.5 & 19.1 & 18.9 & 14 \\
\hline Tristeza & 16.4 & 8.2 & 10.4 & 20.5 & 8.0 & 2.9 & 10.8 & 14.6 \\
\hline Gratitud & 31.1 & 27.4 & 12.5 & 28.9 & 11.6 & 7.4 & 6.8 & 9.1 \\
\hline
\end{tabular}


La tendencia se invierte cuando se trata de la demanda de apoyo. Así,, son los mensajes enviados por mujeres los que incluían con más frecuencia demandas de apoyo informativo y práctico, aunque únicamente en el grupo de asma, $\chi^{2}$ $(1, \mathrm{~N}=131)=0.09, \mathrm{p}<0.001$ y $\chi^{2}(1, \mathrm{~N}=131)=$ $6.04, \mathrm{p}<0.05$, respectivamente. Por último, $\mathrm{y}$ sólo en el grupo de diabetes, también son los mensajes enviados por mujeres los que incluyen más frecuentemente demandas de apoyo emocional, $\chi^{2}(1, \mathrm{~N}=238)=4.94, \mathrm{p}<0.05$.

\section{Discusión}

Los objetivos de este trabajo fueron identificar los tópicos más frecuentes sobre los que se «escribe» en diversos foros de internet sobre problemas de salud en función del grupo diagnóstico y del sexo. Se trata de un trabajo novedoso al ser el primero, hasta nuestro conocimiento, que se dirige a analizar el contenido de los mensajes intercambiados en foros en lengua castellana. Así mismo, se suma a los escasos trabajos que incluyen foros de diferentes grupos diagnósticos (Van Uden-Kraan et al., 2008).

Los resultados indican que la mayoría de los mensajes que se intercambian en estos foros aluden, en primer lugar, a cuestiones relacionadas con la demanda y el ofrecimiento de apoyo $\mathrm{y}$, en segundo lugar, a aspectos de la enfermedad o el tratamiento. El ofrecimiento de apoyo es más frecuente que la demanda del mismo en sus tres dimensiones, informativa, práctica y emocional; en este sentido, es lógico que un mensaje que demanda apoyo movilizará al resto de participantes que irán enlazando mensajes de ofrecimiento de ayuda. Así, la simple inspección visual de la estructura de un foro permite comprobar la existencia de una "hilera» de respuestas múltiples como reacción a un mensaje «raíz» de petición de ayuda. Recientemente hemos comprobado que un $70 \%$ de los mensajes enviados a un foro de padres de niños con diabetes eran de respuesta a otros (Marín, Salaverría, Beléndez y Suriá, 2009).

Nuestros resultados también muestran diferencias significativas en la frecuencia de los temas contenidos en los mensajes en función de la enfermedad. Así, en los foros de asma y diabetes son más frecuentes los mensajes relativos a apoyo práctico e informativo, mientras que en los foros de esclerosis y fibromialgia están más presentes los mensajes que intercambian apoyo emocional. La complejidad del tratamiento de la diabetes que requiere la combinación de inyecciones de insulina, controles de glucemia, ejercicio y dieta explicaría una marcada necesidad de apoyo informativo y práctico. La naturaleza exigente del autocuidado de la diabetes también explicaría la mayor frecuencia de mensajes relativos al tratamiento. Igualmente, las contribuciones al foro de asma se caracterizan por aludir con una alta frecuencia a cuestiones terapéuticas. Al igual que en la diabetes, el tratamiento farmacológico resulta imprescindible para un manejo adecuado de la sintomatología asmática.

Por su parte, la sensación de incomprensión que pueden experimentar las personas aquejadas de fibromialgia, un trastorno de origen desconocido que sólo recientemente empieza a ser considerada como entidad diagnóstica reconocida, puede conducir a la búsqueda de apoyo emocional. Las personas que padecen este síndrome, en ocasiones minimizado por las personas del entorno más próximo y que pueden haber sido objeto de crítica, por ejemplo, en su ámbito laboral, pueden volverse a los foros de internet en busca de otros que comparten síntomas, problemas y dudas similares. Así, la comparación con otras personas en la misma situación satisface la necesidad de validar los síntomas percibidos y reduce el sentimiento de incomprensión (Tennen y Affleck, 1997). El encuentro con otras personas similares con quien compartir sus múltiples e impredecibles dolencias resulta especialmente relevante cuando alguien ha podido sentirse aislado por sentirse diferente a los demás. Este es el caso de algunas personas aquejadas de fibromialgia que, tras muchos años de peregrinaje por consultas de especialistas, reciben finalmente un diagnóstico (Undeland y Malterud, 2007). Ese sentimiento de pertenencia a un grupo y esa identidad compartida podría explicar también la mayor frecuencia de intercambio de mensajes que aluden a emociones positivas de optimismo y gratitud en comparación con el resto de foros.

Respecto a las referencias interpersonales en las contribuciones de los participantes, des- 
tacó el grupo de asma por su frecuencia significativamente superior de alusiones a temas $f a-$ miliares. Parece plausible pensar que dada la imprevisibilidad de las crisis asmáticas la repercusión tanto en el afectado, sobre todo si se trata de niños, como en la familia es evidente (Abásolo, Méndez y Ortigosa, 2003).

El análisis de la frecuencia de los temas de los mensajes en función del sexo muestra que las preferencias de mujeres o varones por unos temas se hacen patentes sólo en algunos tópicos y, únicamente, en los foros de algunas enfermedades. Así, no se hallaron diferencias significativas de género en la frecuencia de alusión a temas relativos a sintomas y tratamiento ni en el contenido emocional en ninguno de los foros de las cuatro enfermedades, con la excepción de la expresión de gratitud (mayor en las mujeres) en el foro de asma.

Sin embargo, las diferencias de género aparecen cuando se analiza la frecuencia de contenidos relativos al ofrecimiento y a la demanda de apoyo. En este sentido, se observó que, en los foros de diabetes y asma, los mensajes enviados por varones incluyeron más referencias de oferta de apoyo informativo y práctico, mientras que los mensajes enviados por mujeres incluyeron con más frecuencia demandas de apoyo informativo y práctico. Por último, y sólo para el caso del foro de diabetes, también son los mensajes enviados por mujeres los que incluyen más frecuentemente demandas de apoyo emocional. Estos resultados son difícilmente comparables con estudios previos que han analizado las diferencias de género en el contenido de los mensajes intercambiados en foros sobre problemas de salud ya que la mayoría de estos trabajos previos han estudiado foros de cáncer (Mo, Malik y Coulson, 2009). No obstante, el hallazgo de diferencias de género sólo en algunas dimensiones y en algunos de los foros estudiados está en la línea de los resultados que los autores de la revisión apuntan en el sentido de que los estudios sobre foros o grupos mixtos, como es nuestro caso, han encontrado que los patrones de comunicación de hombres y mujeres tienden a ser similares. Esta similitud podría ser el resultado de la acomodación o la adaptación de los participantes al estilo de comunicación del género predominante en el foro; de esta forma, sería la composición de varones/mujeres del grupo lo que determina más el estilo de comunicación que el género de los participantes (Savicki y Kelley, 2000). Al mismo tiempo, la falta de señales visuales y el anonimato característico de la interacción virtual puede hacer más saliente la identidad grupal relativa a la enfermedad dejando la identidad grupal de género en un segundo plano.

No obstante, en nuestro trabajo hemos encontrado algunas diferencias exclusivas del caso del intercambio de apoyo. Una posible explicación a la mayor frecuencia de demanda de apoyo en los mensajes enviados por mujeres, en los foros de asma y diabetes, podría deberse a una mayor participación de madres y padres de niños afectados y al hecho de que son las madres las que siguen teniendo el principal papel de responsables del manejo de la enfermedad de sus hijos/as y, por tanto, las que plantearían más dudas sobre el cuidado de la enfermedad (Beléndez, Marín, Lorente, Hurtado y Sanz, 2008).

Aunque el presente trabajo resulta novedoso al ser el primero, hasta nuestro conocimiento, que se dirige a analizar y comparar el contenido de los mensajes intercambiados en distintos foros en lengua castellana, presenta algunas limitaciones que es necesario considerar. Por una parte, se trata de un trabajo exploratorio dirigido al estudio del patrón comunicativo en diferentes foros que analiza el número total de mensajes enviados durante un periodo de tiempo sin tener en cuenta quienes son los emisores de dichos mensajes. Así, no controlamos el número de participantes de los foros pudiendo darse el caso de una sobrerepresentación del estilo de comunicación de unos usuarios, en el caso de diferencias extremas de participación entre ellos. Por otra parte, la mayoría de los foros, excepto el de esclerosis, presenta una mayoría de mensajes procedentes de mujeres. En especial, en el foro de fibromialgia, trastorno frecuentemente diagnosticado en mujeres, el porcentaje de mujeres llega al 70\%. Por último, y debido a la alta heterogeneidad de la composición de este tipo de foros, tampoco fue posible agrupar los mensajes en función del rol del emisor (paciente, familiar, amigo) aunque, como comentamos más arriba, en alguno de los foros existía una proporción importante de mensajes enviados por familiares y, en concreto, madres. 
A pesar de estas limitaciones, nuestro estudio es pionero en el análisis de la comunicación intercambiada en los foros para problemas de salud, un fenómeno que cada vez con más fuerza se está imponiendo como medio para intercambiar información y compartir preocupaciones. Este tipo de estudios poseen especial relevancia a la hora de diseñar intervenciones para pacientes o sus familiares. Así, nos permiten conocer de "primera mano» las creencias, preocupaciones y necesidades de pacientes que, en el anonimato propio de la interacción electrónica, se expresan de manera más libre.

\section{Referencias}

Abásolo, F., Méndez, F. X. y Ortigosa, J. M. (2003). Intervención psicológica en el asma bronquial infantil. En J. M. Ortigosa, M. J. Quiles y F. X. Méndez (Eds.), Manual de Psicología de la salud con niños, adolescentes y familias (pp. 183-196). Madrid: Pirámide.

Anderberg, P. (2007). Peer assistance for personal assistance: Analysis of online discussions about personal assistance from a Swedish Web forum for disabled people. Disability y Society, 22, 251-265.

Bar-Lev, S. (2008). «We are here to give you emotional support»: Performing emotions in an online HIV/AIDS support group. Qualitative Health Research, 18, 509-521.

Barak, A. y Gluck-Ofri, O. (2007). Degree and reciprocity of self-disclosure in online forums. $C y$ berPsychology y Behavior, 10, 407-417.

Beléndez, M., Marín, M.C.; Lorente, I., Hurtado, F.J. y Sanz, O. (November, 2008). Coping strategies and well-being in parents of children with diabetes: gender differences. Presented at the Congress on Therapeutic Patient Education 2008. Budapest, Hungary.

Beléndez, M y Suriá, R. (2008, Noviembre). Promoting patient empowerment via the internet: an analysis of discussion forums for patients with chronic conditions. Póster presentado en Congress on Therapeutic Patient Education. Budapest, Hungría.

Blank, T. O. y Adams-Blodnieks, M. (2007). The who and the what of usage of two cancer online communities. Computers in Human Behavior, 23, 1249-1257.
Braithwaite, D. O., Waldron, V.R. y Finn, J. (1999). Communication of social support in computermediated groups for people with disabilities. Health Communication, 11(2),123-151.

Cline, R. J. W. y Haynes, K. M. (2001). Consumer health information seeking on the Internet: the state of the art. Health Education Research, 16(6), 671-692.

Coulson, N. S. (2005). Receiving social support online: An analysis of a computer-mediated support group for individuals living with irritable Bowel syndrome. CyberPsychology \& Behavior, 8, 580-584.

Dalheim-Englund, A. C., Rydstrom, I., Rasmussen, B. H., Moller, C. y Sandman, P. O. (2004). Having a child with asthma quality of life for Swedish parents. Journal of Clinical Nursing, 13, 386-95.

Davison, K. P. y Pennebaker, J. W. (1997). Virtual narratives: Illness representations in online support groups. En K. J. Petrie y J. Weinman (Eds.), Perceptions of health and illness: Current research and applications (pp 463-486). London: Harwood Academic Press.

Davidson, K., Pennebaker, J. W. y Dickeson, S. (2000). Who talks? The social psychology of illess groups. American Psychologist, 55(2), 205-217.

Demiris, G. (2006). The diffusion of virtual communities in health care: Concepts and challenges. Patient Education and Counseling, 62(2), 178-188.

Eagly, A. H. y Crowley, M. (1986). Gender and helping behaviour: a meta-analytic review of the social psychological literature. Psychological Bulletin, 100 (3), 283-308.

Fallow, D. (2005). How women and men use the Internet. Obtenido 10 de abril, 2007, de Pew Internet y American Life Project. http://www.pewInternet.org/pdfs/PIP_Women_and_Men_online.pdf.

Finn, J. (1999). An exploration of helping processes in an online self-help groupfocusing on issues of disability. Health and Social Work, 24, 220-231.

Ginossar, T. (2008). Online participation: A content analysis of differences in utilization of two online cancer communities by men and women, patients and family members. Health Communication, 23, 1-12.

Gooden, R. J. y Winefield, H. R. (2007). Breast and prostate cancer online discussion boards: a the- 
matic analysis of gender differences and similarities. Journal of Health Psychology, 12, 103-114.

Gray R.E., Fitch M., Davis C. y Phillips C. (1996). Breast cancer and prostate cancer self-help groups: reflections on differences. Psycho-oncology. 5, 137-142.

Klemm, P., Hurst, S. L., Dearholt, S. y Trone, R. (1999). Gender differences on Internet cancer support groups, Computers in Nursing, 17, 65-72.

Lasker, J., Sogolow, D. y Sharim, P. (2005). The role of an online community for people with a rare disease: content analysis of messages posted on a primary biliary cirrhosis mailing list. Journal of Medical Internet Research, 31, 7-10.

Macias, W., Stavchansky, L. y Smith, T. (2005). Health related message boards discussion content and implications for pharmaceutical sponsorships. Journal of Health Communication, 10 (3), 209-223.

Maccoby, E. E. (1990). Gender and relationships: A developmental account. American Psychologist, $45,513-520$.

Marín, M. C., Salaverría, V., Beléndez, M. y Suriá, R. (2009, Octubre). A website about diabetes in children and adolescents: interactive contents and evolution. Comunicación presentada al $20^{\text {th }}$ World Diabetes Congress. Montreal.

Mehl, M. R. y Pennebaker, J. W. (2003). The sounds of social life: A psychometric analysis of students daily social environments and natural conversations. Journal of Personality and Social Psychology, 84, 857-870.

Mo, P. K. H., Sumaira, S. H. y Coulson, N. S. (2009). Gender differences in computer-mediated communication: a systematic literature review of online health-related support groups. Patient Education and Counseling, 75, 16-24.

Moral, F., Canto, J. y Gómez-Jacinto, L. (2004). Internet y desindividuación. Nuevas perspectivas sobre la desindividuación en la red: el modelo de identidad social de los fenómenos de desindividuación (SIDE). Revista de Psicología Social, 19(1), 93-106.

Perron, B. (2002). Online support for caregivers of people with a mental illness. Psychiatric Rehabilitation Journal, 26, 70-77.
Pennebaker, J. W. y King, L. A. (1999). Linguistic styles: Language use as an individual difference. Journal of Personality and Social Psychology, 77, 1296-1312.

Ravert, R. D., Hancock, M. D. y Ingersoll, G. M. (2004). Online forum messages posted by adolescents with type I diabetes. The Diabetes Educator, 30, 27-834.

Rourke, L., Anderson, T., Garrison, D. R., y Archer, W. (2001). Methodological issues in the content analysis of computer conference transcripts. International Journal of Artificial Intelligence in Education, 12, 8-22.

Salem, D. A., Bogat, G. A. y Reid, C. (1997). Mutual help goes on-line. Journal of Community Psychology, 25, 189-207.

Savicki, V., y Kelley, M. (2000). Computer mediated communication: Gender and group composition. CyberPsychology \& Behavior, 3(5), 817-826.

Seale C, Ziebland S, y Charteris-Black, J. (2006). Gender, cancer experience and internet use: A comparative keyword analysis of interviews and online cancer support groups. Social Science and Medicine, 62, 2577-2590.

Sharf, B. F. (1999). Beyond Netiquette: the ethics of doing naturalistic discourseresearch on the Internet. En Jones, S. (Ed.), Doing Internet Research: Critical Issues and Methods for Examining the Net (pp. 243-256). Sage Publications: Newbury Park, CA.

Schultz, P. N., Stava, C., Beck, M. L., y VassilopoulouSellin, R. (2003). Internet message board use by patients with cancer and their families. Clinical Journal of Oncology Nursing, 7, 663-667.

Sullivan, F. C. (2003). Gendered cybersupport: a thematic analysis of two online cancer support groups. Journal of Health Psychology, 8 (1), 83-103.

Suriá, R. y Beléndez, M. (2006, Septiembre). Análisis de los grupos de salud en Internet: un estudio a través del contenido de sus narraciones. Póster presentado en VI Semana de Investigación de la Facultad de Psicología de la UNED. Madrid.

Tennen, H., y Affleck, G. (1997). Social comparison as a coping process: A critical review and application to chronic pain disorders. En B.P. Buunk y F.X. Gibbons, (Eds). Health, coping, and well-being: Perspectives from social comparison theory. 
(pp. 263-298). Mahwah, NJ, US: Lawrence Erlbaum Associates Publishers.

Undeland, M., y Malterud, K. (2007). The fibromyalgia diagnosis-hardly helpful for the patients? Scandinavian Journal of Primary Health Care, 25(4), 250-255.

Van Uden-Kraan C. F., Drossaert C. H. C., Taal E., Lebrun, C. E. I., Drossaers-Bakker, K.W. Smit, W. M., Seydel E. R. y Van de Laar M. A. F. J. (2008). Coping withsomatic illnesses in online support groups. Do the feared disadvantages actually o ccur? Computers in Human Behavior, 24, 309-24.

Walther, J. B., y Boyd, S. (2002). Attraction to computer-mediated social support. En C. A. Lin y D. Atkin (Eds.), Communication technology and so- ciety: Audience adoption and uses (pp. 153-188). Cresskill, NJ: Hampton Press.

Weare, C., y Lin, W. Y. (2000). Content analysis of the world wide web: Opportunities and challenges. Social Science Computer Review, 18(3), 272.

Winefield, R. (2005). Support provision and emotional work in an Internet support group for cancer patients. Health Psychology, 12 (1), 103-114.

Winzelberg, A. J. (1997). The analysis of an electronic support group for individuals with eating disorders. Computers in Human Behavior, 13, 393-407.

Zahn-Waxler, C., Radke-Yarrow, M., Wagner, E. y Chapman, M. (1992). Development of concern for others. Developmental Psychology, 28, 126-136. 